\title{
Emigrantes en el mundo romano: algunas precisiones sobre el término incola
}

\author{
Emigrants in the Roman World: \\ some precisions on the term incola
}

Miguel Ángel NoviLLo LÓPEZ*

\begin{abstract}
RESUMEN
Con el término incola Roma no sólo se refirió a la población indígena de un determinado núcleo que sustraídas sus tierras, generalmente a causa de una fundación colonial no pacífica, era reducida por Roma a tal condición, sino que con la misma denominación Roma agrupó en múltiples ocasiones a la población foránea que desde época republicana se establecía en una comunidad, colonia o municipio, en la que obtenía la condición de residente.

Tomando como referencia los

movimientos migratorios en el mundo romano, en las siguientes páginas abordaremos algunas precisiones conceptuales del término incola desde la segunda de sus acepciones.
\end{abstract}

PALABRAS CLAVE

Movimientos migratorios, estatuto jurídicoadministrativo, incolae, origo, domicilium.

\begin{abstract}
By the term incola, Romans referred not only to the aboriginal population of a concrete place who, once deprived of their land normally through the unpeaceful foundation of a colony, were reduced by Rome to such a condition. Rome also used this term in many occasions to name the foreign population that, already from Republican times, established in a community, colony or municipality, obtaining there their residentship. Taking the migratory trends in the Roman world as a reference, in this paper we will deal with some conceptual aspects of the termincola under its second meaning.
\end{abstract}

\section{KEYWORDS}

Migratory movements, juridicaladministrative statute, incolae, origo, domicilium.

Recibido el 7 de junio de 2012. Aceptado el 5 de agosto de 2012

* Investigador Postdoctoral FECYT, Università degli Studi di Trieste / Dipartimento di Studi Umanistici, Via del Lazzaretto Vecchio 6 - 34123 Trieste, Italia / mnovillo@ units.it 
Durante los dos primeros siglos del Imperio, la romanización alcanzó grandes progresos hasta el punto de que las formas de vida romana se difundieron por todo el ámbito mediterráneo ${ }^{1}$. En este sentido, los movimientos migratorios y las consecuentes estructuras socio-económicas y jurídico-administrativas pueden ser interpretados como cuestiones de crucial importancia para poder comprender mejor el proceso de romanización ${ }^{2}$.

En Roma la definición de extranjero experimentó un proceso de transformación entre la tardía República romana y el año 212 con la aprobación de la constitutio Antoniniana. Los dos principales contextos en los que se desarrolló y articuló la noción de extranjero o trasladado en el mundo romano fueron primordialmente el político-social y el etno-cultural ${ }^{3}$. En este sentido, en las fuentes de la época el extranjero o trasladado podía aparecer citado de diferentes maneras 4 : hospes...

En el ámbito jurídico-político, barbarus, peregrinus, incola, externus, hostis /

En el ámbito étnico-geográfico, numidio, gallus, germanus, aethiops, syrus, aibraios, iudaeus...

En el ámbito somático, fuscus, indus, maurus, coloratus, ager, niger, albus, candidus...

${ }^{1}$ Bajo Claudio, pero sobre todo desde los Flavios, se incrementó considerablemente el número de ciudadanos romanos extraídos de los peregrini. Además, a las fundaciones coloniales de Julio César y de Augusto siguió la creación de nuevos municipios durante las primeras dinastías y de colonias militares por todo el Imperio hasta bien entrado el gobierno de Adriano.

${ }^{2}$ En este sentido, no hay que olvidar que el verdadero objetivo de la actuación romana consistió en reorganizar a la población de la forma más eficaz, tanto para la explotación de los recursos como de la propia población. Véanse PRIETO, Alberto, «El espacio social del poder», Studia Storica, 20, 2002, pp. 139-170; RODAZZ, Jean Michel, «De la conquête a la pacification: la mutation des societés indigènes», en RODRÍGUEZ NEILA, Juan Francisco (ed.), Sociedad y economía en el Occidente romano. Pamplona, 2003, pp. 15-27.

${ }^{3}$ Conviene señalar que la mayoría de los individuos de origen occidental presentan una onomástica latinizada o helenizada correspondiente a la categoría social más que a la identidad étnica. Precisamente la onomástica es uno de los agentes sobre los que se apoya el estudio de la emigración, ya que su testimonio es un criterio comúnmente empleado, en ausencia de origo, para identificar la presencia de población foránea. En este sentido, son dos los criterios principales que se emplean para explicar la difusión onomástica: la imitatio y la clientela. GARCÍA FERNÁNDEZ, Estela, «Movilidad, onomástica e integración en Hispania en época republicana: Algunas observaciones metodológicas», en IGLESIAS GIL, José Manuel y RUIZ GUTIÉRREZ, Alicia (eds.), Viajes y cambios de residencia en el mundo romano. Santander, 2011, pp. 47-66, esp. pp. 48-56.

${ }^{4}$ Véanse GAUDEMET, Jean, «L'étranger dans le monde romain», Studii Clasice, 7, 1965, pp. 37-47; $\mathrm{RICCI}$, Cecilia, Orbis in urbe. Fenomeni migratory nella Roma imperiale. Roma, 2005, esp. pp. 6-8; HERMON, Ella, "Des communautés distinctes sur la même territoire. Quelle fut la réalité des incolae?», en COMPATANGELO-SOUSSIGNAN, Rita y SCHWENTZEL, Christian Georges (eds.), Etrangers dans la cité romaine. Actes du Colloque de Valenciennes (14 - 15 octobre 2005). Rennes, 2007, pp. 25-42, esp. pp. 39-40. C. MIHAILESCU-BÎRLIBA, Lucretio - DUMITRACHE, Lulia - PIFTOR, Valentin, «La mobilitè locale en Dacie Romaine selon les sources épigraphiques: I. Dacie inférieure», Studia historica. Historia Antigua, 28, 2010, pp. 95-108. NOVILLO LÓPEZ, Miguel Ángel, «Movimenti migratori e modeli di gestione territoriale e clientelare in Transpadana: alcuni aspetti metodologici» en MAGNANI, Stefano (ed.), Tra l'Adriatico e le Alpi: forme e sviluppi dell'organizzazione territoriale e dei procesi de integrazione nella $x$ regio orientale e nelle regioni contermini. Udine, en prensa 2013. 
Bajo el término incola ${ }^{5}$ Roma no sólo agrupó a la población foránea ${ }^{6}$ que desde época tardorrepublicana se establecía individual y libremente en una comunidad, colonia o municipio, en la que obtenía la condición de residente ${ }^{7}$, sino que en variadas ocasiones con tal denominación hacía referencia a la población indígena de un determinado núcleo que despojada de sus tierras, como norma general a causa de una fundación colonial no pacífica, era reducida por Roma a tal condición ${ }^{8}$. Es decir, en el interior de la categoría de incola puede existir una duplicidad de componentes: por un lado aquellos que, a título individual y a causa de diversas circunstancias, han dejado su ciudad de origen y se han establecido en otro territorio cívico, y son por consiguiente forasteros domiciliados ${ }^{9}$, y por otro lado aquellos que, como población indígena ${ }^{10}$, han experimentado un proceso de colonización en su territorio y se someten a convivir con aquellos que han llegado permaneciendo en áreas no centuriadas o en áreas no asignadas ${ }^{11}$.

\footnotetext{
${ }^{5}$ Varias son las acepciones del término incola en su significado común. En primer lugar, el término comenzó a emplearse en las descripciones geográficas para referirse a aquellos pueblos que habitaban una región concreta del mundo (Plin., NH., 2.70.180; 3.3.25; 3.5.71; 3.9.94; 3.20.133; 3.20.134; 4.7.27; Cic., Nat. Deor., 2.56.140; Liv., 21.38; 22.46; 27.29; 28.3). En latín el término incola tiene la primera acepción de habitante de un territorio. Existe una estrecha relación entre los términos incola y colonus, si bien el primero hace referencia a los habitantes indígenas de un determinado territorio, mientras que el segundo alude al colectivo romano que tomaba posesión del territorio sustrayendo sus tierras. El término incola también guarda relación con el término accola que igualmente puede traducirse como habitante, si bien era aquel que habitaba en los alrededores. Véanse PAPAZOGLOU, Fanoula, Laoi et paroikoi. Recherches sur les structures de la sociétéhellénistique, Beograd, 1997, p. 234; LE ROUX, Patrick, «Peregrini Incolae», Zeitschrift für Papyrologie und Epigraphik, 154, 2005, pp. 261-266, esp. p. 265.

${ }^{6}$ Además de movimiento humano extraprovincial hay que tener presente el movimiento humano interprovincial.

${ }^{7}$ Por otro lado se encontrarían aquellas personas que indicaban la recepción de la ciudadanía local, lo que podía traer consigo una permanencia anterior como incolae en esa misma comunidad.

${ }^{8}$ Dentro del conjunto de los incolae indígenas es necesario distinguir dos grupos: en primer lugar el formado por los incolae en sentido estricto, es decir, aquellos que genera la propia comunidad promocionada, y en segundo lugar el integrado por la población indígena que en numerosas ocasiones Roma incorpora a sus fundaciones coloniales después de haber extinguido jurídica y administrativamente la antigua ciudadanía indígena. Véanse RODRIGUEZ NEILA, Juan Francisco, «La situación socio-política de los incolae en el mundo romano», Memorias de Historia Antigua, 2, 1978, pp. 147-169; GARCíA FERNÁNDEZ, Estela, «Incolae contributi y la lex Ursonensis», Studia historica. Historia Antigua, 15, 1997, pp. 171-180, esp. pp. 173 y 176.

${ }^{9}$ En época republicana si los incolae que habían fijado su domicilium en otra ciudad distinta de su origo no eran completamente miembros de la misma y su cambio de residencia no derogaba su pertenencia a su ciudad de origen, del mismo modo los romanos en una ciudad peregrina seguían siendo romanos, ellos y sus descendientes, y los peregrinos en una ciudad romana continuaban siendo peregrinos ellos y sus ascendientes. CALZADA GONZÁLEZ, Aránzazu, «Origo, incolae, municipes y civitas romana a la luz de la lex Irnitana», Revista Internacional de Derecho Romano, 4, 2010, pp. 17-51, esp. p. 45.

${ }^{10}$ La presencia de incolae indígenas no se registra en todas las colonias. Las fuentes gromáticas del siglo I atestiguan claramente que cuando los indígenas de un territorio no eran sometidos o expulsados, en tal caso los indígenas eran considerados incolae. En este sentido, se distinguían claramente de los colonos porque, mientras que estos últimos poseían tria nomina, los incolae indígenas tan sólo contaba con su nombre seguido del patronímico. Los incolae indígenas estuvieron presentes en las colonias latinas fundadas entre los siglos IV-II a.C. y en las colonias romanas hasta la aprobación de la constitutio Antoniniana. Véanse CÁSSOLA, Filippo y LABRUNA, Luigi, Lineamenti di storia del diritto romano. Milano, 1989, pp. 246 ss.; GAGLIARDI, Lorenzo, Mobilità e integrazione delle persone nei centri cittadini romani. Aspetti giuridici. I. La classificazione degli incolae. Milano, 2006, p. 20.

${ }^{11}$ Los incolae no formaban parte del populus, es decir, se encontraban al margen de dicha realidad ciudadana por más que pudiesen participar en la vida política de la ciudad.
} 
En las fuentes clásicas contamos con tres definiciones 'en positivo' del término incola. La primera corresponde al jurista Sexto Pomponio ${ }^{12}$, para quien el término incola estaba estrechamente ligado al término domicilium. Por consiguiente, calificaba como incola a aquella persona que adoptaba el domicilium del lugar donde se había trasladado siendo diferente su origo. Asimismo, según el jurista eran incolae tanto aquellos que fijaban su domicilio en el núcleo de una colonia o de un municipio como aquellos que se establecían en el territorio circundante de dichos núcleos sin ser municipes o coloni ${ }^{13}$. Así pues, los incolae eran en definitiva los residentes trasladados, es decir, los individuos que trasladaban su propio domicilio a una nueva ciudad o territorio - el que dicho colectivo residiera en comunidades distintas a sus lugares de origen, en las que no tenían derechos políticos ni ciudadanía reconocida, no opta para que se haga constar en ocasiones su condición de residentes-. Podemos añadir, además, que no necesariamente debía tratarse de ciudadanos romanos, afirmación que es también compatible con la inmigración de peregrinos que se trasladaban de un lugar a otro ${ }^{14}$. Por tanto, los incolae indígenas no pueden ser definidos como aquellos individuos que cambiaban su domicilio. $\mathrm{Pa}$ ralelamente, Agustín de Hipona ${ }^{15}$ define al incola como aquella persona que se encontraba en una ciudad distinta, es decir, aquella persona que simplemente se encontraba fuera de su patria, si bien los define como inmigrantes permanentes. En último lugar, para Isidoro de Sevilla ${ }^{16}$ el incola era aquel inmigrante que establecía su domicilio con carácter permanente en un nuevo centro cívico. De esta manera, en los tres autores la noción de incola se encuentra estrechamente ligada a la de traslado ${ }^{17}$.

Cada vez que encontremos en las fuentes el término incola tendremos que tratar de comprender a qué tipo de incolae se refiere, si indígenas o trasladados ${ }^{18}$, si bien es cierto que a partir del siglo II y hasta bien entrado el siglo III en el registro

12 DIG., 50.16.239.2.

${ }^{13}$ KUHN, Emil, Die städtische und bürgerliche Verfassung des römischen Reichs bis auf die Zeiten Justinians. Leipzig, 2011, p. 31.

${ }^{14}$ Abordar los movimientos migratorios es una cuestión que presenta serias dificultades debido a que la documentación existente es bastante escasa y ambigua y, además, no existen cifras que permitan realizar valoraciones cuantitativas. Véanse WILSON, Alan John Nisbet, Emigration from Italy in the Republican Age of Rome. New York, 1966; HALEY, Evan W., Migration and economy in Roman Imperial Spain. Barcelona, 1991.

${ }^{15}$ Cfr. Sen., Epist., 70.16.

${ }^{16}$ Isid., Etymol., 9.4.37-40.

17 Asimismo en el capítulo 98 de la lex Ursonensis se deduce que los incolae eran un grupo de domiciliados / residentes que se diferenciaban claramente de los colonos. Por otro lado, como ya apuntase Calzada González, la alusión a los incolae en la lex Irnitana se refiere sin lugar a dudas a los individuos de distinta origo instalados en Irni, si bien su origo no era obstáculo para quedar igualmente sujetos a la ley municipal como si fueran ellos mismos municipes implicando así una asimilación de personas con anterior adscripción a otras ciudades a los irnitatos, que va más allá de los derechos de los latinos en Roma durante el siglo I a.C., pues estos aun habiendo adquirido la civitas romana per migrationem et censum, podían ser expulsados volviendo a ostentar de esta manera la civitas de su primera origo. CALZADA GONZÁLEZ, Aránzazu, Op. Cit., 2010, p. 38.

${ }_{18}$ Podemos afirmar que no existen razones suficientes como para sostener que cuando las fuentes mencionan a los incolae excluyan directamente a los indígenas. 
epigráfico comenzarán a atestiguarse individuos peregrini llamados incolae ${ }^{19}$. A partir del siglo III el término incolae se empleó mayoritariamente para designar a los individuos de un municipio o de una colonia domiciliados en otro municipio o colonia. En este sentido, es muy probable que Agustín de Hipona o Isidoro de Sevilla, autores que escribieron en época posterior a la fecha de la constitutio Antoniniana, ignorasen por completo la noción de incola indígena al emplear tan sólo la de incola trasladado.

La presencia de los incolae trasladados tanto en Italia ${ }^{20}$ como en las colonias y en los municipios provinciales es realmente difusa, pues en realidad representan un colectivo con aspectos bastante ambiguos e ininteligibles en el cuadro administrativo y social del mundo romano ${ }^{21}$.

En los repertorios epigráficos los incolae se nos manifiestan desde una perspectiva fundamentalmente jurídica. No obstante, el término y la realidad vivida por los incolae no se extinguen en las formulaciones jurídicas presentes en el registro epigráfico, sino que por el contrario en su sentido no jurídico los incolae aparecen en numerosas ocasiones en las fuentes históricas haciendo referencia a una realidad no jurídica ${ }^{22}$.

Como norma general, los incolae presentes en cualquier civitas pueden presentar un variado estatuto, es decir, pueden aparecer como ciudadanos romanos con diversa origo ${ }^{23}$, como colonos latinos o como peregrinos — no sólo hay que analizar la origo con relación a la civitas romana, pues de hecho un número notable de ciudadanos romanos se desplazaban fuera de su ciudad a una colonia lati-

${ }^{19}$ CHASTAGNOL, André, «Coloni et incolae. Notes sur les différenciations sociales à l'intérieur des colonies romaines de pleuplement dans les provinces de l'Occident (ler siècele av. J.-C. - ler siècle ap. J.-C.)», Splendidissima civitas. Hommage François Jacques. París, 1996, pp. 13-25.

${ }^{20}$ MROZEK, Stanislaw, «Quelques observations sur les incolae en Italie», Epigraphica, 46, 1984, pp. 17-21.

${ }^{21}$ Contamos con evidencias epigráficas suficientes que confirman la dispersión del concepto de incola trasladado desde época republicana. No obstante, resulta demasiado complicado cuantificar los testimonios literarios de los incolae trasladados, pues es mínima la información totalmente cierta y numerosas las referencias colectivas. El indicio principal para la individualización de los incolae trasladados es la presencia de etnónimos, si bien otros criterios de individualización son los epítetos, los atributos somáticos o las referencias de pertenencia a asociaciones étnicas o religiosas. Véanse BERGER, Adolf, «Incola», en Pauly-Wissowa, IX, 2. Stuttgart, 1916, coll. 1249-1255; DE MARTINO, Francesco, Storia della costituzione romana, III. Napoli, 1961, pp. 254 ss.; LAFFI, Umberto, Adtributio e Contributio. Problemi del sistema politico-amministrativo dello stato romano. Pisa, 1966, pp. 193-208; TILLY, Charles, «The historical Study of vital Processes», en VV.AA., Historical Studies of Changing Fertility. Princeton, 1978, pp. 3-56; PORTILLO MARTÍN, Rafael, Incolae. Una contribución al análisis de la movilidad social en el mundo romano. Córdoba, 1983; POMA, Gabriella, «Incolae: alcune osservazioni», Rivista Storica dell'Antichità, 28, 1988, pp. 135-147; THOMAS, Yan, Origine et Commune Patrie. Étude de droit public romain (89 av. J.-C.-212 ap. J.-C.). Rome, 1996; GAGLIARDI, Lorenzo, Op. Cit., 2006.

${ }^{22}$ Donde mejor se observa el carácter no jurídico del término incola es en las fuentes literarias. LOMAS SALMONTE, Francisco Javier, «De la condición social de los incolae con especial referencia a Hispania», Habis, 18, 1987, pp. 383-395, esp. p. 384.

${ }^{23}$ Es necesario apuntar que la origo tan sólo adquiere relevancia técnica a partir de Adriano, y que desde entonces tuvo un valor sistemático y sobre todo implicaciones fiscales. NÖRR, D, «Origo. Studien zur Orts-, Stadt- und Reichsangehörigkeit in der Antike», Tijdschrift voor Rechtsgeschiedenis, 31, 1963, pp. 525-600. 
na, a un municipio, a una civitas foederata y a la inversa; los habitantes de otras ciudades de las que traían su origo se trasladaban a Roma o a las colonias y municipios civium romani o bien iuris Latini, estableciendo nuevos vínculos con las ciudades en las que se asentaban de manera permanente o a las que emigraban ${ }^{24}$.

El domicilium representaría el vínculo jurídico que determinaba la condición del incola hacia la comunidad a la que emigraba ${ }^{25}$.

Desde una perspectiva sociopolítica ${ }^{26}$, en las fuentes clásicas el término incola designa a quien, perteneciendo por su origo y ciudadanía local a una determinada comunidad, se instalaba en otra fijando en ella su nuevo domicilium ${ }^{27}$. Por consiguiente, no se trataba de ciudadanos, sino de residentes, siendo el domicilio el único elemento que determinaba la condición del incola ${ }^{28}$. Empero, la condición

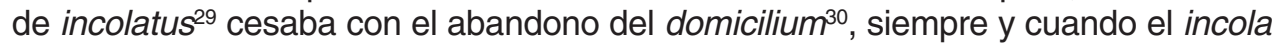
hubiese hecho frente a todas sus cargas o munera que, como norma general, podía imponerle la ciudad que le había acogido ${ }^{31}$. En ésta no adquiría la condición de ciudadano, sino que conservaba, de manera conjunta con sus descendientes, los derechos de ciudadanía inherentes a su origo. En la comunidad donde establecía su nueva residencia el incola quedaba sometido a varios deberes otorgándosele en ciertas ocasiones algunos derechos. Como ya señalase Rodríguez Nei-

${ }^{24}$ CALZADA GONZÁLEZ, Aránzazu, Op. Cit., 2010, p. 31.

${ }^{25} \mathrm{El}$ domicilium designa el lugar de residencia, por lo que es un vínculo jurídico entre la ciudad y la persona que ha emigrado a ella. En este sentido, la epigrafía jurídica tardorrepublicana marca claramente la distinción entre los ciudadanos propiamente dichos y los incolae. Véanse ZILLETTI, Ugo, «Incolato», NNDI, 8, 1962, pp. 541-542; GONZÁLEZ FERNÁNDEZ, Rafael y MOLINA GÓMEZ, José Antonio, «Precisiones a las menciones de origo con la fórmula domo + topónimo / gentilicio en la epigrafía romana de Hispania», Emerita. Revista de Lingüística y Filología Clásica, 79.1, 2011, pp. 1-29, esp. p. 1.

${ }^{26}$ RODRÍGUEZ NEILA, Juan Francisco, Op. It., 1978, pp. 147-169.

${ }_{27}$ Incolae no sólo eran los domiciliados venidos de fuera que se establecían en el casco urbano, es decir, los incolae intramurani, sino que también lo eran aquellos que se instalaban dentro de los límites del territorium, esto es, los incolae extramurani, si bien es cierto que, en clave pragmática, existían diferencias notables entre unos y otros en cuanto a participación cívica se refiere. GAGLIARDI, Lorenzo, "Osservazioni in tema di domicilio degli 'incolae'. La distinzione tra 'incolae' di città e 'incolae' di campagna», en CAPOGROSSI COLOGNESI, Luigi y GABBA, Emilio (eds.), Gli Statuti Municipali. Pavia, 2006, pp. 647-672.

${ }_{28}$ Un individuo podía ser domiciliado en el lugar donde se establecía la sede de los negocios, donde se instalaban los lares familiares o donde se poseían los bienes patrimoniales. No era suficiente con una simple permanencia eventual o con poseer tan sólo un inmueble. Los requisitos constitutivos del domicilio comportaban que, con carácter general, éste fuese único. Sin embargo, se acabó aceptando que, de manera excepcional, un individuo pudiera tener varios domicilios cuando estuviera establecido por igual en más de un lugar. Y, del mismo modo, con el mismo carácter excepcional se admitió que pudiera carecer de domicilio cuando, habiendo abandonado el actual, se encontrase buscando otro o se dedicase a viajar durante un largo periodo. Véanse, Tabula Heraclensis, Ins. 157-158; DIG., 50.1.5; 47.10.5; LANGHAMMER Walter, Die rechtliche und soziale Stellung der 'Magistratus municipales' und der 'Decuriones'. Wiesbaden, 1973, p. 29; LÓPEZ HUGUET, María Luisa, «El domicilio de las personas jurídicas: evolución desde el Derecho romano y significado actual», REDUR, 6, diciembre 2008, pp. 69-94, esp. p. 72.

${ }_{29}$ LICANDRO, Orazio, «Domicilium e incolae tra Repubblica e Principato», en COMPATANGELOSOUSSIGNAN, Rita y SCHWENTZEL, Christian Georges (eds.), Etrangers dans la cité romaine. Actes du Colloque de Valenciennes (14 - 15 octobre 2005). Rennes, 2007, pp. 43-76.

30 DIG., 50.1.20.

${ }^{31}$ DIG., 50.1.17.11; 50.1.20.34; Cod. lust., 10, 40, 1; Cod. Theod., 12.1.52. 
la, puesto que los incolae constituían dentro de una ciudad un sector característico de la población, son la consideración de su condición política, social y económica, así como el análisis de las obligaciones que recaían sobre ellos, los elementos que permiten concretar cuál fue su situación real en la ciudad.

Thomas $^{32}$ distingue de una parte a los ciudadanos de pleno derecho, es decir, cives, coloni, y municipes y de otra parte a los incolae, que interpreta simplemente como el conjunto de extranjeros residentes en la ciudad de acogida. En este sentido, la gran división entre cives e incolae tiene claramente un sentido local, si bien es cierto que queda indefinido si no se relaciona con la ciudadanía romana.

Por otro lado, un problema que es necesario abordar es el relacionado con la procedencia de los incolae trasladados. En este sentido, la documentación epigráfica manifiesta el carácter extrínseco respecto a la ciudadanía local que tiene el sector de incolae de una determinada comunidad que les viene asignado por proceder de otras ciudades y no poseer la ciudadanía en aquellas donde establecen su domicilium con carácter temporal o definitivo. La diferencia, como ya señalase Rodríguez Neila, viene manifiesta en las inscripciones, cuando se citan separadamente el cuerpo de ciudadanos y el de incolae ${ }^{33}$. La diferencia de los incolae respecto a los cives hay que entenderla en cuanto a la disposición o no de la totalidad de los derechos y deberes inherentes a la ciudadanía local. Pero el que los incolae sean citados en segundo término tiene su lógica pues, sin presentar los derechos de los ciudadanos originales, integrados en la civitas por filiación real o ficticia los incolae pasan a estar relativamente asimilados al cuerpo social de cada ciudad. Y no sólo por el hecho de fijar en ella un domicilium, que puede ser compartido con el que se tiene en la localidad de origen, sino en virtud de determinados mecanismos de integración que los acercan a la situación político-administrativa o social de los verdaderos cives. Los testimonios epigráficos alusivos a los incolae, permiten sostener que en la mayoría de los casos conformaban un sector definido de la población -empero, la relativa abundancia de testimonios en los que aparecen citados como cuerpo político-administrativo o sector social contrasta con los pocos ejemplos existentes de individuos que dejan constancia de su condición de incola, si bien es cierto que son varios los ejemplos en los que el incola menciona su condición de ciudadano en su ciudad de origen con el único objeto de resaltar el orgullo del origen personal- ${ }^{34}$. No obstante, los testimonios epigráficos en los que se alude directamente a la categoría de incola son tan limitados, que no es posible confirmar con detalle las rutas de desplazamiento.

32 Tanto cives como coloni y municipes eran ciudadanos de pleno derecho de su comunidad, y a través de su ciudad pertenecían paralelamente a la patria común. THOMAS, Yan, Op. Cit., 1996, pp. 13.

${ }^{33}$ RODRÍGUEZ NEILA, J.F., Op.Cit., 1978, p. 148.

${ }^{34}$ El estudio de los movimientos migratorios en el mundo romano implica la búsqueda en el registro epigráfico de aquellos individuos que fueron incolae pero no dejaron una referencia explícita de tal condición. Este análisis ha de tomar como punto de referencia aquellas inscripciones donde aparecen individuos con una origo diferente a la ciudad donde toman el domicilium o que recogen directamente la recepción de cualquier ciudadanía local. 
El volumen demográfico puede variar en función de varias razones: circunstancias especiales en el proceso de creación de una colonia, la realidad económica de la ciudad, existencia en los estatutos administrativos de medidas en favor de los incolae, etc. Es decir, se trata de factores que pueden favorecer o no la movilidad de determinados sectores de la población forzados a trasladar su domicilium a otro lugar por razones de índole económica o social ${ }^{35}$.

Los incolae estaban también sometidos a las leyes locales de su ciudad de origen y a las leyes locales de su ciudad de residencia. Asimismo, no perdían la vinculación local determinada por su origo y permanecían, en consecuencia, cives, peregrini, municipes o coloni de derecho romano o latino. No obstante, no contamos con los datos suficientes que permitan confirmar si el concepto de incola es tan poco flexible como para igualar en un mismo nivel de derechos y deberes a individuos que, siendo muy diferentes entre sí, coinciden en el hecho de haber fijado su domicilium en una determinada ciudad sin haber accedido a la ciudadanía local.

Por último es necesario señalar que los incolae, fuesen ciudadanos romanos o latinos, o bien simples peregrinos, por no disfrutar de la ciudadanía local allí donde habían instalado su nuevo domicilio, quedaban al margen de los principales derechos políticos, si bien quedaban sujetos a la jurisdicción de los magistrados de su localidad de residencia, pero disponían, asimismo, de los iura políticos en sus comunidades de origen y se les respetaban los privilegios propios de su rango, posición y derechos civiles personales ${ }^{36}$.

${ }^{35}$ Preferentemente quien migraba lo hacía sin familia o antes de tenerla con el único objeto de conseguir un mayor número de oportunidades de trabajo. No obstante, entre los motivos de las migraciones también figuran los relacionados con la formación del individuo, el correcto desarrollo de la carrera política o militar, el desarrollo de cualquier actividad profesional o el reencuentro familiar. Roma empleaba un término general y técnico para definir a aquellos que se trasladaban por motivos comerciales: negotiatiores. Sin embargo, no todos los negotiatiores eran incolae trasladados, pues con dicho término se designaban fundamentalmente a los mercaderes o a los individuos que permanecían de manera eventual en una ciudad. Una vez que se habían asentado de manera permanente eran llamados consistentes, término que designaba genéricamente a los residentes y que no tenía, a su vez, un empleo jurídico técnico. Véanse CHEVALLIER, Raymond, Voyages et déplacements dans l'Empire romain. París, 1988; MOATTI, Claudia (ed.), La mobilité des personnes en Méditerranée de l'Antiquité à l'époque moderne. Procédures de contrôle et documents d'identification, Rome, 2004; GAGLIARDI, Lorenzo, Op. Cit., 2006, p. 50 ss.

${ }^{36}$ A diferencia de los municipes, los incolae no podían ser elegibles para los puestos rectores locales. Aunque en las inscripciones aparezcan en numerosas ocasiones equiparados a los cives, en varias ocasiones existen distinciones que reflejan la situación y estima de cada sector dentro de la escala social. Los incolae podían poseer propiedades en el territorio municipal o colonial, si bien es cierto que la sola posesión de terrenos o de una vivienda no era suficiente para basar razonablemente el domicilio (DIG., 50.1.16; Cid. lust., 10.40(38).4). 